\title{
Synthesis of the Taxol Core via Catalytic Asymmetric 1,4-Addition of an Alkylzirconium Nucleophile
}

\author{
Jiao Yu Joseph Wang, Stephen P. Fletcher*
}

Chemistry Research Laboratory, University of Oxford, 12 Mansfield Road, Oxford OX1 3TA, U.K. Supporting Information Placeholder

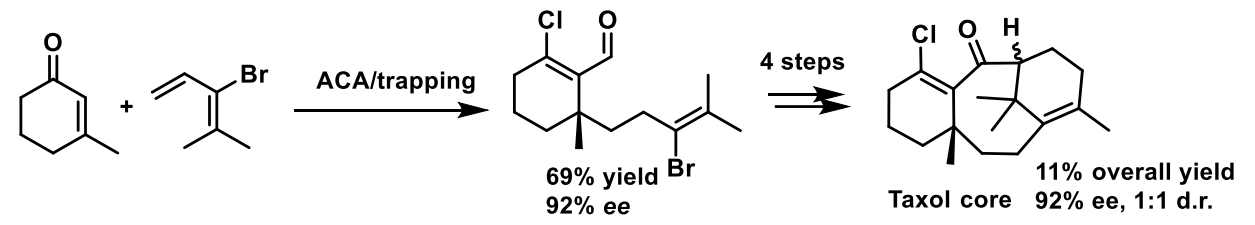

\begin{abstract}
The Taxol core was prepared in five steps via a key copper-catalyzed asymmetric conjugate addition (ACA) trapping sequence. The use of a bromodiene derived alkylzirconium nucleophile followed by trapping with $\mathrm{POCl}_{3} / \mathrm{DMF}$ give a highly functionalized intermediate featuring a quaternary centre in $69 \%$ yield and $92 \%$ ee. After 1,2-addition, Suzuki-Miyaura cross coupling, allylic oxidation, and a type II intramolecular Diels-Alder reaction the taxol core was obtained in $11 \%$ overall yield with $92 \%$ ee.
\end{abstract}

Taxol 1 (trademarked as Paclitaxel) is a multi-billion dollar anticancer drug. In the past, interest in this molecule stemmed from solving an issue of supply; Taxol was only available in appreciable quantities from the Yew tree and harvesting from such a source was not sustainable. ${ }^{\text {a }}$ A significant effort was devoted toward the chemical synthesis of $\mathbf{1}$ and many advances in organic chemistry were made by groups attempting to solve this challenging problem. ${ }^{1 \mathrm{~b}-\mathrm{j}}$

Scheme 1. A) Synthesis of (+)-Taxadiene by Baran and coworkers B) This work

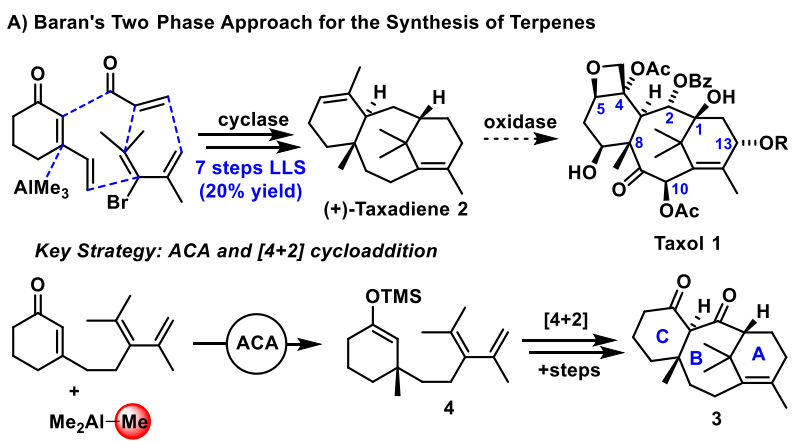

B) This work: Can we perform ACAs with more elaborate nucleophiles?

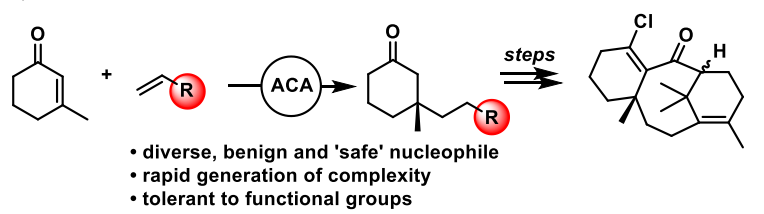

A recent strategy for the synthesis of terpenes proposed by Baran and co-workers involves first constructing the carbonbased framework of the molecule, followed by the installation of functional groups via a series of late stage oxidations to give the target. ${ }^{2 \mathrm{a}-\mathrm{c}}$ Using this approach, oxidized Taxanes including Taxol itself were prepared by the combination of a "cyclase" phase to form the carbon skeleton, followed by an "oxidase" phase to adjust the final oxidation state of the molecule (Scheme 1, A). ${ }^{2 \mathrm{~d}-\mathrm{e}}$ Thus, the cyclase phase was implemented to provide Taxadiene $\mathbf{2}$ in 7 steps and 20\% overall yield. The brevity in the synthesis of $\mathbf{2}$ arises from two key features (Scheme 1, B); the use of a Type II intramolecular Diels-Alder reaction to prepare the A/B rings of 3, a strategy shared by Shea and others to prepare Taxane derivatives, ${ }^{3}$ and a catalytic asymmetric conjugate addition (ACA) for the formation of $\mathbf{4}$ using Alexakis's protocol for Me-addition. ${ }^{4}$

The latter of these tactics was the main inspiration for this project and we wondered if we could add fragments more complex than simple alkyl units such as methyl (Scheme 1, C). The nucleophiles traditionally used in ACAs (organozinc, organoaluminum and Grignard reagents) ${ }^{5}$ are limited to only very simple coupling partners. Further, the use of these organometallic reagents imposes limits on which functional groups can be used and the applicability of these procedures in complex molecule synthesis. In 2013, we reported the formation of quaternary stereogenic centers ${ }^{6}$ by ACA of alkylzirconium reagents $^{7}$ to enones. ${ }^{8}$ The transformations are catalyzed by copper-phosphoramidite complexes and the alkyl zirconocenes are generated in situ from alkenes and $\mathrm{Cp}_{2} \mathrm{ZrHCl}$ via hydrometallation. Alkylzirconium reagents, when used in this fashion, can mitigate the aforementioned shortcomings of traditional nucleophiles since they are more tolerant towards functional groups. We hoped to use an appropriate fragment in a synthesis of the taxol core but the precise choice of the precursor alkene was not immediately obvious. Also, it is more common than not, that ACAs are sensitive to solvent, tempera- 
ture, concentration, method of addition and presence of additives. $^{5}$

In an approach that would have closely follow Baran's route to the core, conjugated triene $5^{9}$ was added to 6 (Scheme 2). Despite extensive optimization, 7 could only be obtained in $14 \%$ yield and $80 \%$ ee. Although disappointing, no side products that would result from hydrozirconation at the di- or tetrasubstituted olefins were observed and we postulated that the low yield was due to the size of the polyene.

Scheme 2. Preparation of Intermediate 7 using ACA of Triene 5

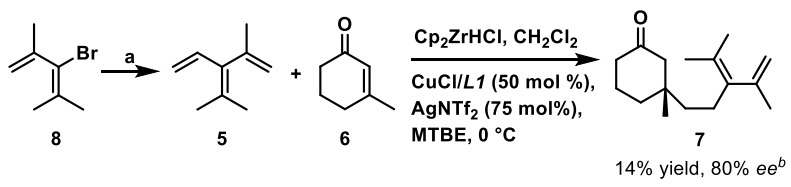

a) Vinylmagnesium bromide (1.3 eq.), $\mathrm{ZnBr}_{2}$ (2.0 eq.), $\mathrm{PdCl}_{2}$.dppf (0.020 eq.), THF $0{ }^{\circ} \mathrm{C}$ to rt $6.5 \mathrm{~h}, 49 \%$; b) ee determined by SFC or HPLC. Abbreviations: $\operatorname{dppf}=1,1$ 'Bis(diphenylphosphino)ferrocene

In our search for an alkene with a smaller steric profile, we encountered bromodiene $\mathbf{9}^{10}$ first reported by Takahashi and co-workers. 9 was prepared from 3-methylcrotonaldehyde via bromination to the corresponding bromoaldehyde, followed by Peterson olefination and could easily be made on a decagram scale (see supporting information for more details).

Table 1. Optimisation of Key ACA Conjugate Addition Step

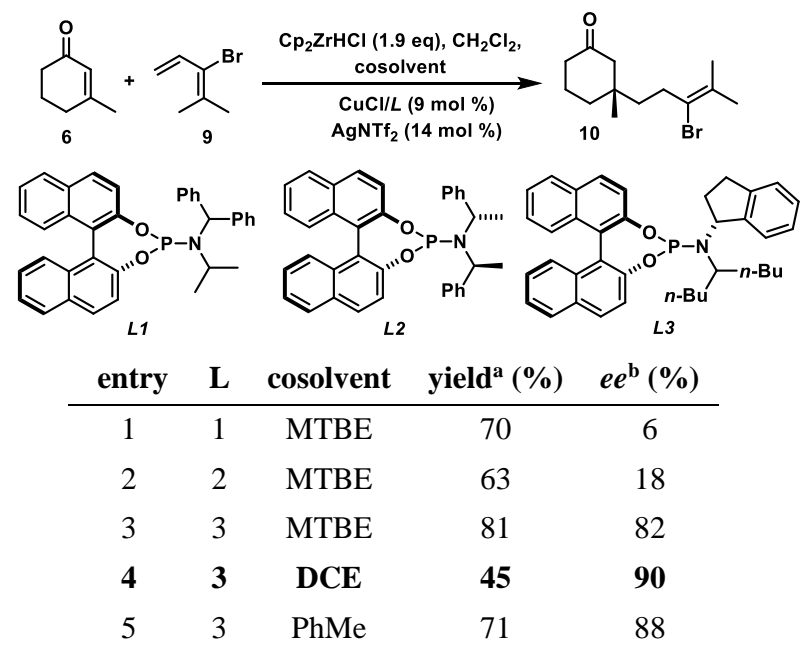

*Reactions were performed on $0.5 \mathrm{mmol}$ scale using 2.4 eq 9 . a) isolated yields; b) ee's were determined by SFC or HPLC analysis using a chiral non-racemic stationary phase.

The ACA of 9 to $\mathbf{6}$ was examined and it was found that the phosphoramidite ligand has a tremendous effect on enantioselectivity of this reaction (Table 1). L1 and $\mathbf{L} \mathbf{2}$ have both been used extensively in similar transformations ${ }^{6 a, 7}$ but gave poor enantioselectivities for our system. To our delight, $\mathbf{L 3},{ }^{\text {a }}$ developed in our group and used in other challenging transformations, ${ }^{8 \mathrm{e}, 11-13}$ was found to give the best yield/ee combination and is easily prepared. Investigation of different counterions $\left(\mathrm{BF}_{4}, \mathrm{SbF}_{6}, \mathrm{OTf}, \mathrm{PF}_{6}, \mathrm{ClO}_{4}\right)$ indicated that $\mathrm{NTf}_{2}$ gave superior yields and enantioselectivity. Previous experiments in the group showed that using dichloromethane as a solvent was often beneficial. The use of a chlorinated cosolvent gave better enantioselectivity but lower yields. With hydrocarbon or ethereal cosolvents, higher yields but lower ee's were observed.

Upon scale-up (to $3 \mathrm{mmol}$ of $\mathbf{6}$ ) the reaction performed better, and the product could be obtained in $96 \%$ yield and $88 \%$ ee. ACAs of alkylzirconium nucleophiles often work better when scaled up; this effect is attributed to the fact that it is easier to measure and mix the reaction components at larger scales, and larger scales minimize the impact of trace air and moisture.

Having demonstrated that 9 could reliably be added to $\mathbf{6}$, we next turned our attention to the preparation of a suitable intermediate for the synthesis of the desired product. The trapping of zirconium enolates is a challenging problem, ${ }^{14}$ and we recently reported trapping reactions using the Vilsmeier-Haack reagent to give $\beta$-chloroaldehydes from our ACA zirconium enolates. ${ }^{15}$ It was found that this trapping protocol also worked for our substrate; using $16.5 \mathrm{mmol}$ of $\mathbf{6}, 3.6$ grams of $\mathbf{1 1}$ (corresponding to $69 \%$ yield) could be obtained in $92 \%$ ee requiring minimal modification (see supporting information for optimization) (Scheme 3, steps a-b).

Scheme 3. Completion of the Synthesis

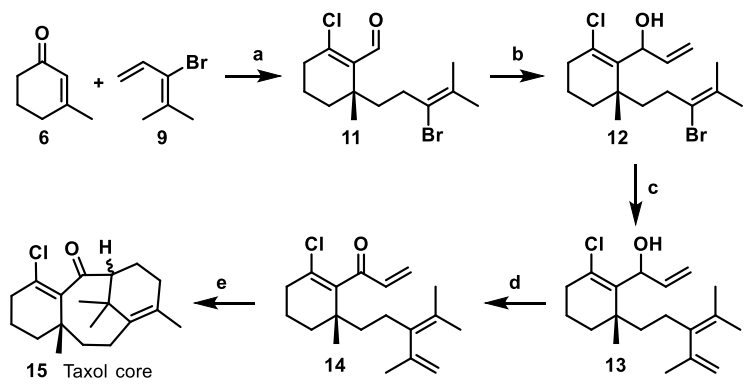

a) $\mathrm{Cp}_{2} \mathrm{ZrHCl}$ (1.9 eq), $\mathrm{CuCl} / \mathrm{L3}$ (0.08 eq), $\mathrm{DCE} / \mathrm{CH}_{2} \mathrm{Cl}_{2}$ rt 17 hrs; then $\mathrm{POCl}_{3} / \mathrm{DMF}$ (10 eq), DCE $60{ }^{\circ} \mathrm{C} 1 \mathrm{~h}, 69 \%$, 92\% ee; b) vinylmagnesium bromide (1.2 eq), $\mathrm{Et}_{2} \mathrm{O} 0{ }^{\circ} \mathrm{C} 1 \mathrm{~h}, 87 \%$; c) isopropenylboronic acid pinacol ester $(1.1 \mathrm{eq}), \mathrm{PdCl}_{2}\left(\mathrm{PPh}_{3}\right)_{2} / \mathrm{dppf}(0.10$ eq), $\mathrm{K}_{3} \mathrm{PO}_{4}\left(3.0\right.$ eq), DMF $65{ }^{\circ} \mathrm{C} 17 \mathrm{~h}, 59 \%$; d) Dess-Martin periodinane (1.2 eq), $\mathrm{H}_{2} \mathrm{O}(0.50 \mathrm{eq}), \mathrm{CH}_{2} \mathrm{Cl}_{2}$ rt $30 \mathrm{~min}$ undesired:desired $=1: 10,90 \%$; e) $\mathrm{TiCl}_{4}(1.1 \mathrm{eq})$ slow addition, $\mathrm{CH}_{2} \mathrm{Cl}_{2}-35 \quad{ }^{\circ} \mathrm{C} \quad 6 \quad \mathrm{~h}, \quad 35 \%, \quad 1: 1 \quad$ d.r $92 \%$ ee, Abbreviations: $\quad D C E=1,2$-dichloroethane, $\operatorname{dppf}=1,1$ 'Bis(diphenylphosphino)ferrocene.

To complete the synthesis, allylic alcohol 12 was obtained from 1,2-addition of vinylmagnesium bromide to 11 . Then, standard Suzuki-Miyaura conditions with isopropenyl boronic acid pinacol ester gave triene $\mathbf{1 3}$ in $59 \%$ yield. ${ }^{16}$ The final two steps of the synthesis proved to be problematic. Triene $\mathbf{1 3}$ was found to be unstable under various oxidation conditions (see supporting information). Eventually, it was found that the use of Dess-Martin periodinane with one equivalent of water gave good results. Without water an allylic transposition product was observed in appreciable quantities. ${ }^{17}$

For the Diels-Alder reaction, we investigated a number of different conditions spanning both thermal and Lewis acid promoted transformations (see supporting information). Although the conditions reported by Baran and co-workers (3.65 eq. $\mathrm{BF}_{3} \cdot \mathrm{Et}_{2} \mathrm{O}$, slow addition at $\left.0{ }^{\circ} \mathrm{C}\right)^{2 \mathrm{a}}$ gave trace product $(10 \%$ 
yield, 1:1 d.r., 92\% ee) our substrate - being structurally different to that employed by Baran and co-workers - required extensive screening in order to reach an acceptable yield. The best results were obtained via slow addition of the substrate to a dilute and cold $\left(-35^{\circ} \mathrm{C}\right)$ solution of the Lewis acid which furnished $\mathbf{1 5}$ in $35 \%$ yield as a 1:1 mixture of diastereomers at $\mathrm{C} 1$ and $92 \%$ ee.

One might assume that quaternary stereocenter $\mathrm{C} 8$ is responsible for stereoinduction at $\mathrm{C} 1$ and that poor diastereoselectivity is a result of the remoteness of C8. In the Diels-Alder reaction to form 2, Baran and co-workers observed a diastereomeric ratio of 2:1 (desired:undesired) at $\mathrm{C} 3$ in the cyclisation precursor which corresponded to an identical ratio at $\mathrm{C} 1$ in the product. ${ }^{2 \mathrm{a}}$ While these centers are $\alpha$ to carbonyls, this may suggest that $\mathrm{C} 3$ is responsible for the diastereoselectivity, and we note that $\mathbf{1 4}$ lacks a $\mathrm{C} 3$ stereocenter.

In conclusion, the Taxol core was prepared in five steps from commercially available 3-methyl-2-cyclohex-2-ene-1one 6 in $11 \%$ yield and $92 \%$ ee. The key step employed a hydrometallation ACA/trapping sequence of functionalized alkene 9 to furnish unsaturated $\beta$-chloroaldehyde $\mathbf{1 1}$ in high enantiomeric excess. Further elaboration of this intermediate to the ketone was accomplished via an addition, cross coupling and oxidation sequence. Finally, an intramolecular Diels-Alder reaction simultaneously formed the $\mathrm{A}$ and $\mathrm{B}$ rings to deliver the Taxol core. We believe that this work highlights the benefits of using organozirconium nucleophiles in asymmetric addition reactions for total synthesis and related applications.

\section{ASSOCIATED CONTENT}

\section{Supporting Information}

The Supporting Information is available free of charge on the ACS Publications website.

All procedures, characterization data, NMR spectra, and chromatography traces (PDF).

\section{AUTHOR INFORMATION}

\section{Corresponding Author}

*E-mail: stephen.fletcher@chem.ox.ac.uk

\section{ORCID}

Stephen P. Fletcher: 0000-0001-7629-0997

Jiao Yu Joseph Wang: 0000-0002-6180-7452

\section{Notes}

The authors declare no competing financial interests.

\section{ACKNOWLEDGMENT}

We thank Thomas Palacin (University of Oxford) and Mireira Sidera (University of Oxford) for initial studies, and the EPSRC EP/H003711/1 for funding.

\section{REFERENCES}

(1) a) Hudlicky, T.; Reed, J. W. The Way of Synthesis : Evolution of Design and Methods for Natural Products; Wiley-VCH: Weinheim, Germany, 2007. For syntheses of Taxol see: b) Holton, R. A.; Kim, H. B.; Somoza, C.; Liang, F.; Biediger, R. J.; Boatman, P. D.; Shindo, M.; Smith, C. C.; Kim, S.; Nadizadeh, H.; Suzuki, Y.; Tao, C.; Vu, P.; Tang, S.; Zhang, P.; Murthi, K. K.; Gentile, L. N.; Liu, J. H. First total synthesis of taxol. 2. Completion of the $\mathrm{C}$ and D Rings. J. Am. Chem. Soc. 1994, 116, 1599-1600. c) Nicolaou, K. C.; Yang, Z.; Liu, J. J.; Ueno, H.;
Nantermet, P. G.; Guy, R. K.; Claiborne, C. F.; Renaud, J.; Couladouros, E. A.; Paulvannan, K.; Sorensen, E. J. Total synthesis of taxol. Nature 1994, 367, 630-634. d) Danishefsky, S. J.; Masters, J. J.; Young, W. B.; Link, J. T.; Snyder, L. B.; Magee, T. V.; Jung, D. K.; Isaacs, R. C. A.; Bornmann, W. G.; Alaimo, C. A.; Coburn, C. A.; Di Grandi, M. J. Total synthesis of baccatin III and taxol. J. Am. Chem. Soc. 1996, 118, 28432859. e) Wender, P. A.; Badham, N. F.; Conway, S. P.; Floreancig, P. E.; Glass, T. E.; Houze, J. B.; Krauss, N. E.; Lee, D.; Marquess, D. G.; McGrane, P. L.; Meng, W.; Natchus, M. G.; Shuker, A. J.; Sutton, J. C.; Taylor, R. E. The pinene path to taxanes. 6. A concise stereocontrolled synthesis of taxol. J. Am. Chem. Soc. 1997, 119, 2757-2758. f) Mukaiyama, T.; Shiina, I.; Iwadare, H.; Saitoh, M.; Nishimura, T.; Ohkawa, N.; Sakoh, H.; Nishimura, K.; Tani, Y.; Hasegawa, M.; Yamada, K.; Saitoh, K. Asymmetric total synthesis of Taxol. Chem. Eur. J. 1999, 5, 121-161. g) Kusama, H.; Hara, R.; Kawahara, S.; Nishimori, T.; Kashima, H.; Nakamura, N.; Morihira, K.; Kuwajima, I. Enantioselective Total Synthesis of (-) -Taxol. J. Am. Chem. Soc. 2000, 112, 3811-3820. h) Doi, T.; Fuse, S.; Miyamoto, S.; Nakai, K.; Sasuga, D.; Takahashi, T. A formal total synthesis of taxol aided by an automated synthesizer. Chem. - An Asian J. 2006, 1, 370-383. i) Hirai, S.; Utsugi, M.; Iwamoto, M.; Nakada, M. Formal total synthesis of (-)-taxol through Pdcatalyzed eight-membered carbocyclic ring formation. Chem. - A Eur. J. 2015, 21, 355-359. j) Fukaya, K.; Kodama, K.; Tanaka, Y.; Yamazaki, H.; Sugai, T.; Yamaguchi, Y.; Watanabe, A.; Oishi, T.; Sato, T.; Chida, N. Synthesis of paclitaxel. 2. construction of the ABCD ring and formal synthesis. Org. Lett. 2015, 17, 2574-2577.

(2) Synthesis of Taxadiene and two-phase approach of terpene synthesis: a) Mendoza, A.; Ishihara, Y.; Baran, P. S. Scalable enantioselective total synthesis of taxanes. Nat. Chem. 2012, 4, 21-25. b) Ishihara, Y.; Mendoza, A.; Baran, P. S. Total synthesis of taxane terpenes: Cyclase phase. Tetrahedron 2013, 69, 5685-5701. c) Krasutsky, S. G.; Jacobo, S. H.; Tweedie, S. R.; Krishnamoorthy, R.; Filatov, A. S. Route optimization and synthesis of taxadienone. Org. Proc. Res. Dev. 2015, 19, 284-289. d) Ishihara, Y.; Baran, P. S. Two-phase terpene total synthesis: Historical perspective and application to the Taxol problem. Synlett 2010, 1733-1745. e) Kanda, Y.; Nakamura, H.; Umemiya, S.; Puthukanoori, R. K.; Appala, V. R. M.; Gaddamanugu, G. K.; Paraselli, B. R.; Baran, P. Two-Phase Synthesis of Taxol®. 2020. https://dx.doi.org/10.26434/CHEMRXIV.12061620.V1.

(3) Selected examples of intramolecular Diels-Alder reactions used in Taxane syntheses: a) Sakan, K.; Craven, B. M. Synthetic Studies on the Taxane Diterpenes. Utility of the Intramolecular Diels-Alder Reaction for a Single-Step Stereocontrolled Synthesis of a Taxane Model System. J. Am. Chem. Soc. 1983, 105, 3732-3734. b) Bonnert, R. V; Jenkins, P. R. A Synthesis of an Alkylated Taxane Model System. $J$. Chem. Soc Perkin Trans. 1 1989, 413-418. c) Yadav, J. S. Synthesis of antitumour agents. Pure Appl. Chem. 1993, 65, 1349-1356. d) Winkler, J. D.; Kim, H. S.; Kim, S. A highly efficient synthesis of taxanes via the tandem Diels-Alder reaction. Tetrahedron Lett. 1995, 36, 687-690. e) Rubenstein, S. M.; Williams, R. M. Studies on the Biosynthesis of Taxol: Total Synthesis of Taxa-4(20),11(12)-diene and Taxa4(5),11(12)-diene. The First Committed Biosynthetic Intermediate. J. Org. Chem. 1995, 60, 7215-7223. f) Stec, M. M.; Gwaltney, S. L.; Burke, L. D.; Nguyen, H.; Shea, K. J. Stereoselective synthesis of an advanced taxusin intermediate: An application of the type 2 intramolecular Diels-Alder reaction. Tetrahedron Lett. 2003, 44, 9379-9382.

(4) Vuagnoux-D’Augustin, M.; Alexakis, A. Copper-catalyzed asymmetric conjugate addition of trialkylaluminium reagents to trisubstituted enones: Construction of chiral quaternary centers. Chem. A Eur. J. 2007, 13, 9647-9662.

(5) a) Jerphagnon, T.; Pizzuti, M. G.; Minnaard, A. J.; Feringa, B. L. Recent advances in enantioselective copper-catalyzed 1,4-addition. Chem. Soc. Rev. 2009, 38, 1039-1075. b) Maksymowicz, R. M.; Bissette, A. J.; Fletcher, S. P. Asymmetric conjugate additions and allylic alkylations using nucleophiles generated by hydro- or carbometallation. Chem. Eur. J. 2015, 21, 5668-5678.

(6) Asymmetric conjugate addition to form quaternary centers: a) Sidera, M.; Roth, P. M. C.; Maksymowicz, R. M.; Fletcher, S. P. Formation of quaternary centers by copper-catalyzed asymmetric conju- 
gate addition of alkylzirconium reagents. Angew. Chemie - Int. Ed. 2013, 52, 7995-7999. b) Roth, P. M. C.; Sidera, M.; Maksymowicz, R. M.; Fletcher, S. P. Copper-catalyzed asymmetric conjugate addition of alkylzirconium reagents to cyclic enones to form quaternary centers. Nat. Protoc. 2014, 9, 104-111. Acyclic systems: c) Gao, Z.; Fletcher, S. P. Acyclic quaternary centers from asymmetric conjugate addition of alkylzirconium reagents to linear trisubstituted enones. Chem. Sci. 2017, 8, 641-646. Five-membered rings: d) Ardkhean, R.; Mortimore, M.; Paton, R. S.; Fletcher, S. P. Formation of quaternary centres by copper catalysed asymmetric conjugate addition to $\beta$ substituted cyclopentenones with the aid of a quantitative structureselectivity relationship. Chem. Sci. 2018, 9, 2628-2632.

(7) Maksymowicz, R. M.; Roth, P. M. C.; Fletcher, S. P. Catalytic asymmetric carbon-carbon bond formation using alkenes as alkylmetal equivalents. Nat. Chem. 2012, 4, 649-654.

(8) For the use of this approach in complex molecule synthesis see a) Wu, J.; Kadonaga, Y.; Hong, B.; Wang, J.; Lei, X. Enantioselective Total Synthesis of (+)-Jungermatrobrunin A. Angew. Chemie - Int. Ed. 2019, 58, 10879-10883. b) Wang, J.; Hong, B.; Hu, D.; Kadonaga, Y.; Tang, R.; Lei, X. Protecting-Group-Free Syntheses of ent-Kaurane Diterpenoids: $[3+2+1]$ Cycloaddition/Cycloalkenylation Approach. $J$. Am. Chem. Soc. 2020, 142, 2238-2243. c) Caprioglio, D.; Fletcher, S. $\mathrm{P}$. An alternative synthesis of the breast cancer drug fulvestrant (Faslodex ${ }^{\circledR}$ ): catalyst control over $\mathrm{C}-\mathrm{C}$ bond formation. Chem. Commun. 2015, 51, 14866-14868. d) Garrec, K.; Fletcher, S. P. Cp2ZrMeCl: A Reagent for Asymmetric Methyl Addition. Org. Lett. 2016, 18, 3814-3817. e) Gao, Z.; Fletcher, S. P. Asymmetric conjugate addition of alkylzirconium reagents to $\alpha, \beta$-unsaturated thioesters: Access to fragrances and acyclic stereochemical arrays. Chem. Commun. 2017, 53, 10216-10219.

(9) Triene $\mathbf{5}$ was obtained in one step via Negishi cross-coupling of vinyl zinc bromide and bromodiene $\mathbf{8}$ in $49 \%$ yield. Bromodiene 8 precursor: a) Jackson, R. W.; Shea, K. J. Synthesis of a C-1 epi taxinine intermediate using the type 2 intramolecular Diels-Alder approach. Tetrahedron Lett. 1994, 35, 1317-1320. b) Magnus, P.; Westwood, N.; Spyvee, M.; Frost, C.; Linnane, P.; Tavares, F.; Lynch, V. Taxane diterpenes 5: Synthesis of the A- and C-rings: An unusual rearrangement of an $\mathrm{N}$-hydroxyimino lactone. Tetrahedron 1999, 55, 6435-6452. c) Laurent, A.; Villalva-Servín, N. P.; Forgione, P.; Wilson, P. D.; Smil, D. V; Fallis, A. G. Part 1: Efficient strategies for the construction of variably substituted bicyclo[5.3.1] undecenones (AB taxane ring systems). Can. J. Chem. 2004, $82,215-226$.

(10) Ogasawara, M.; Ge, Y.; Uetake, K.; Fan, L.; Takahashi, T. Preparation of multisubstituted allenes from allylsilanes. J. Org. Chem. 2005, 70, 3871-3876.

(11) Gao, Z.; Fletcher, S. P. Construction of $\beta$ to carbonyl stereogenic centres by asymmetric 1,4-addition of alkylzirconocenes to dienones and ynenones. Chem. Commun. 2018, 54, 3601-3604.

(12) Brethomé, A. V.; Paton, R. S.; Fletcher, S. P. Retooling Asymmetric Conjugate Additions for Sterically Demanding Substrates with an Iterative Data-Driven Approach. ACS Catal. 2019, 9, 7179-7187.

(13) Jacques, R.; Pullin, R. D. C.; Fletcher, S. P. Desymmetrization of meso-bisphosphates using copper catalysis and alkylzirconocene nucleophiles. Nat. Commun. 2019, 10, 1-6.

(14) a) Némethová, I.; Sorádová, Z.; Šebesta, R. Electrophilic Trapping of Zirconium Enolates Obtained by Copper-Catalyzed Addition of In Situ Generated Organozirconium Reagents. Synthesis 2017 49, 2461-2469. b) Vargová, D.; Némethová, I.; Plevová, K.; Šebesta, R. Asymmetric Transition-Metal Catalysis in the Formation and Functionalization of Metal Enolates. ACS Catal. 2019, 9, 3104-3143.

(15) Wang, J. Y. J.; Palacin, T.; Fletcher, S. P. $\beta$-Chloroaldehydes from Trapping Zirconium Enolates Produced in Asymmetric 1,4Additions. Org. Lett. 2019, 21, 378-381.

(16) The yields could not be improved and we attributed this to the steric hindrance at the bromide.
(17) Luzzio, F. A. 1,3-Oxidative transpositions of allylic alcohols in organic synthesis. Tetrahedron 2012, 68, 5323-5339. 